\title{
Imaging Human Bone Marrow Stem Cell Morphogenesis in Polyglycolic Acid Scaffold by Multiphoton Microscopy
}

\author{
HSUAN-SHU LEE, M.D., ${ }^{1,4}$ SHU-WEN TENG, B.Sc., ${ }^{2}$ HSIAO-CHING CHEN, M.S., ${ }^{1}$ \\ WEN LO, M.S., ${ }^{2}$ YEN SUN, M.S., ${ }^{2}$ TZE-YU LIN, M.S., ${ }^{2}$ LING-LING CHIOU, B.Sc, ${ }^{1}$ \\ CHING-CHUAN JIANG, M.D., ${ }^{3}$ and CHEN-YUAN DONG, Ph.D. ${ }^{2}$
}

\begin{abstract}
The noninvasive imaging of tissue engineering constructs is vital for understanding the physiological changes in construct formation and the design of improved products for therapeutic purposes. In this work, we use the combination of multiphoton autofluorescence and second harmonic generation (SHG) microscopy to image the physiological changes to the engineered constructs of human mesenchymal stem cells seeded in a polyglycolic acid (PGA) scaffold under induction by chondrogenic transforming growth factor-ß3. Without histological procedures, we found that multiphoton autofluorescence is useful for imaging the PGA scaffold and stem cells while SHG is useful for following the progress of extracellular matrix (ECM) formation. We found that the initial ECM formation tends to align along the PGA scaffold orientation and progressive induction alters the scaffold conformation, indicating that biomechanical forces or the chemical environment generated by chondrogenesis is sufficient for scaffold reorganization. Our results suggest that in the future this approach may be used for real-time monitoring of the physiological processes associated with tissue engineering.
\end{abstract}

\section{INTRODUCTION}

T ISSUE ENGINEERING IS AN EMERGING BIOTECHNOLOGY aiming to engineer various tissues in vitro for transplanting into the human body to replace damaged or degenerated tissues or organs. ${ }^{1}$ Cartilage is one of the most actively investigated tissues in the field. However, as in many areas of medicine, the conventional approaches used to monitor the end-product of tissues (e.g., cartilage) are through either histological analyses by fixation, sectioning, and staining or biochemical analyses via extraction and determination of various constituents. ${ }^{2}$ Although such approaches are useful in providing morphological information of the engineered products, a number of drawbacks exist. First, these approaches correspond to the end of sample vitality, and physiologically dynamic processes cannot be followed using such approaches. In addition, the interaction of engineered tissues with native tissues under in vivo conditions cannot be investigated easily.

For a successful transplantation surgery, a demand for noninvasive and nondestructive methods to assess the quality of the engineered tissues or organs before surgical transplantation is extremely useful for the understanding of tissue physiology leading to the improvement of engineered tissues.

A promising technique that has not been widespread in the imaging of engineered tissues is multiphoton microscopy. In this approach, a fluorescent molecule that is typically excited by the absorption of an ultraviolet (UV) or visible photon is excited by the simultaneous absorption of 2 or more nearinfrared photons. Confocal-like imaging quality, reduced photodamage, enhanced imaging depths, and the convenient

Departments of ${ }^{1}$ Internal Medicine and ${ }^{3}$ Orthopedics, National Taiwan University Hospital and National Taiwan University, College of Medicine, Taipei, Taiwan, R.O.C.

${ }^{2}$ Department of Physics and ${ }^{4}$ Institute of Biotechnology, College of Bioresources and Agriculture, National Taiwan University, Taipei, Taiwan, R.O.C. 
access of the entire emission spectrum are the key advantages of the multiphoton approach. ${ }^{3,4}$ In addition to nonlinear fluorescence excitation, the nonlinear polarization effect of second harmonic generation ( $\mathrm{SHG}$ ) has also proven to be a powerful technique for imaging noncentrosymmetric biological structures such as the collagen matrix. ${ }^{5,6}$ Although multiphoton fluorescence microscopy has been applied for tissue engineering studies, to the best of our knowledge, the combination of multiphoton autofluorescence (AF) and SHG imaging in the monitoring of stem cell morphogenesis in a scaffold has not been attempted. ${ }^{7,8}$

Bone marrow (BM) mesenchymal stem cells (MSCs) are multilineage potential cells capable of differentiating into bone, cartilage, and fat cells when induced by appropriate biological cues. ${ }^{9,10}$ These cells have been shown to be able to differentiate into cartilage-like tissues when they were cultured in 3-dimensional scaffolds with induction by biological stimuli, most importantly transformation growth factor (TGF). ${ }^{11,12}$ In this study, we engineered cartilage tissues in vitro using human MSCs seeded on 3-dimensional polyglycolic acid (PGA)-based scaffolds and under induction with factors including TGF- $\beta 3$. Multiphoton AF and SHG imaging of the engineered tissues were performed at regular intervals for up to 3 weeks after chondrogenic induction.

\section{MATERIALS AND METHODS}

\section{Derivation and culture expansion of BM-derived MSCs}

Human BM were obtained and culture expansion of MSCs was performed as previously described. ${ }^{13}$ In brief, with informed consent from patients undergoing total hip replacement, 5-10 $\mathrm{mL}$ of $\mathrm{BM}$ was drawn from proximal femur bones during surgery into $10-\mathrm{mL}$ syringes rinsed with heparin. The BMs were then transferred to $50-\mathrm{mL}$ conical tubes. Whenever present, bone chips in the BM were removed after brief sedimentation by gravity. The fat component was removed by adequate rounds of washing the BMs with phosphate-buffered saline (PBS) and centrifuging at $500 \times g$ for $10 \mathrm{~min}$. Finally, the BM cells were suspended in Dulbecco's modified Eagle's medium-low glucose (DMEMLG, GIBCO, Grand Island, NY) supplemented with $10 \%$ fetal bovine serum (FBS, HyClone, Logan, UT), $10 \mathrm{U} / \mathrm{mL}$ of penicillin $\mathrm{G}$, and $10 \mu \mathrm{g} / \mathrm{mL}$ of streptomycin (GIBCO) and cultured in $75-\mathrm{cm}^{2}$ flasks. The medium was changed every 3-4 days. Nonadherent marrow cells, including red blood cells, were removed during medium changes. The leaving adherent fibroblastic MSCs were expanded in the same medium until passage 7 when they were used for this study.

\section{Cartilaginous tissue engineering}

Nonwoven scaffolds, $2.3 \mathrm{~mm}$ thick, spun with fibers of PGA, were purchased from Synthecon (Houston, TX). They were cut into $3.5 \times 3.5 \mathrm{~mm}$ pieces. The expanded MSCs were trypsinized and resuspended in original medium at a concentration of $2 \times 10^{7}$ cells $/ \mathrm{mL}$. Both sides of the scaffold were statically seeded consecutively with $20 \mu \mathrm{L}$ of the cell suspension. The last side for cell seeding was kept on top (thus designated as "top side") during the subsequent culture. The scaffolds containing cells were then cultured in the medium for 1 week, and followed by chondrogenic induction in serum-free DMEM containing $1 \times \mathrm{ITS}^{+}$Premix (BD Biosciences, Bedford, MA), $0.1 \mathrm{mM}$ of L-ascorbic acid-2phosphate, $10^{-7} \mathrm{M}$ of dexamethasone, and $10 \mathrm{ng} / \mathrm{mL}$ of recombinant human TGF- $\beta 3$ (R\&D Systems, Minneapolis, $\mathrm{MN}$ ) for up to 3 weeks. The chondrogenic induction medium was replaced every 3-4 days. Immediately before and 1, 2, and 3 weeks after chondrogenic induction (W0, W1, W2, and $\mathrm{W} 3$, respectively), one of the engineered tissues was used for RNA extraction, and another for multiphoton microscopic examination before it was embedded in OCT compound (ThermoShandon, Pittsburgh, PA) for further sectioning for immunohistochemistry studies.

\section{Multiphoton AF and SHG microscopy}

To induce multiphoton AF and SHG signals from the engineered tissues, the output of a titanium-sapphire laser (Tsunami, Spectra Physics, Mountain View, CA) pumped by a diode-pumped solid-state laser (Millennia X, Spectra Physics) was used as the excitation source. The $760 \mathrm{~nm}$ output of the laser system is scanned via a $x-y$ mirror scanning system (Model 6220, Cambridge Technology, Cambridge, MA) and guided toward a modified commercial upright microscope (E800, Nikon, Japan). To expand the excitation source, the input of the microscope accommodates a beam expander. The expanded laser is reflected into the back aperture of the objective (Plan Fluor 20×, NA 0.75, Nikon) by a primary dichroic mirror (700DCSPXRUV-3p, Chroma Technology, Rockingham, VT). The power at the sample is approximately $26 \mathrm{~mW}$ and sample luminescence is collected in the epi-illuminated geometry. After passing through the primary dichroic mirror, the AF and SHG signals are separated into 2 detector channels by a secondary dichroic mirror (435DCXP, Chroma Technology). The AF and SHG signals are further processed by 2 band-pass filters centered at 490 (HQ490/40, Chroma Technology) and 380 nm (HQ380/40, Chroma Technology), respectively. The detection bandwidths of both filters are $\pm 20 \mathrm{~nm}$. Singlephoton counting photomultiplier tubes (R7400P, Hamamatsu, Japan) were used as optical detectors. Image acquisition was achieved via both optical and mechanical means. Each optical scan is composed of $256 \times 256$ pixels and each small area optical scan takes about $23 \mathrm{~s}$ to complete. To acquire a large area map of the engineered tissues, the specimen was translated using a mechanical stage (H101, Prior Scientific, UK) to an adjacent position of the specimen after each optical scan, and each large area image is composed of a $2 \times 2$ array of the small area images. In all cases, the 

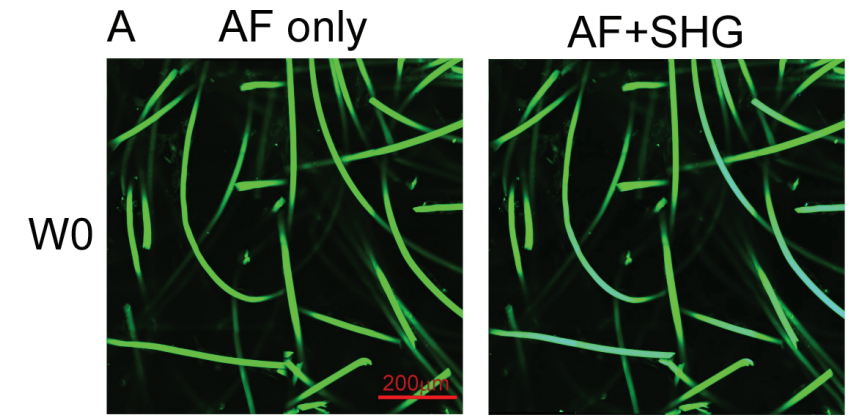

B
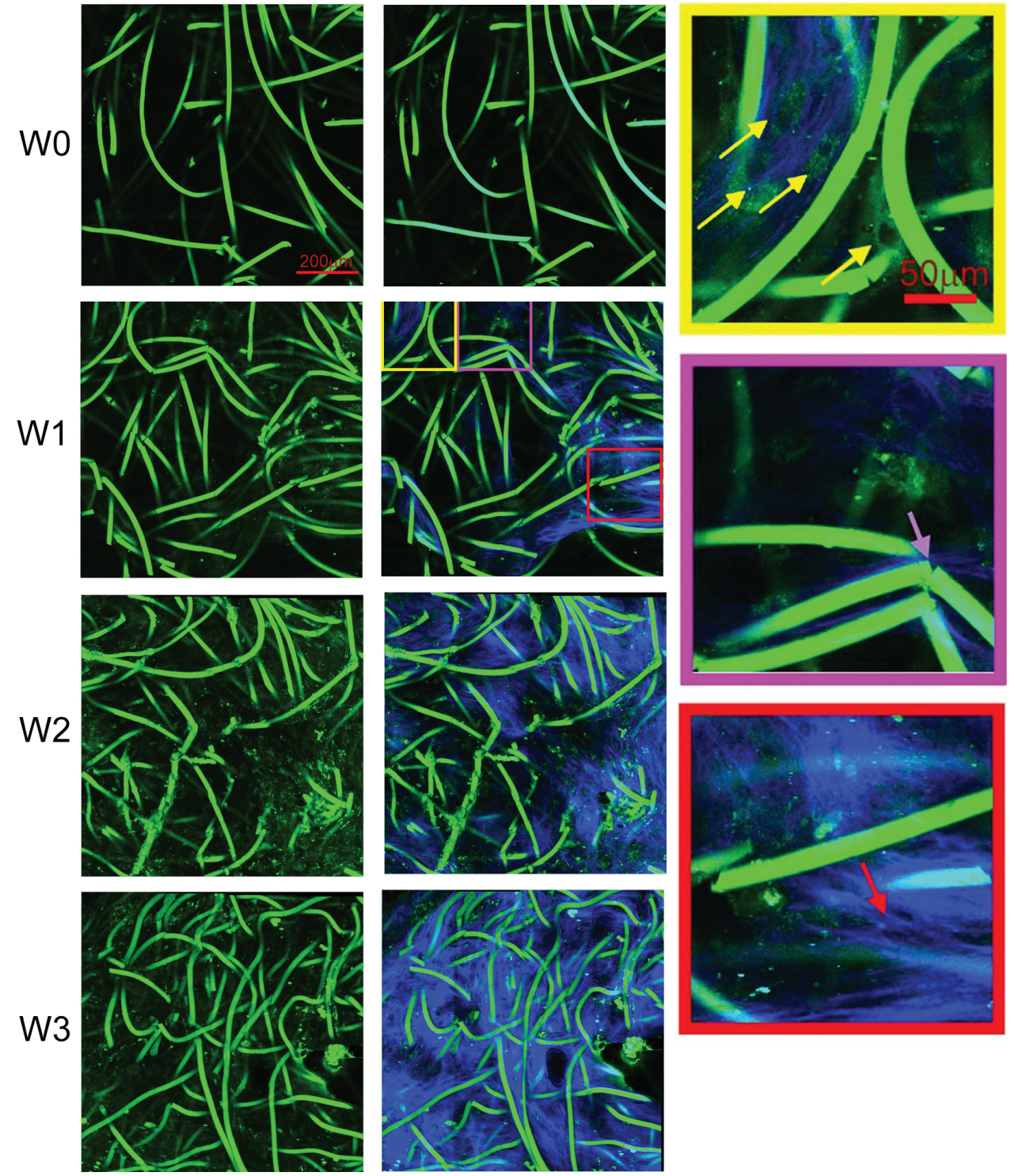

FIG. 1. Multiphoton autofluorescence (AF, in green) and second harmonic generation (SHG, in blue) imaging of engineered cartilage (in PGA scaffold) at different times. Shown on the right panel in A are the combined AF and SHG images and shown on the left are the AF-only results. The images were acquired (from top to bottom) at W0, W1, W2, and W3 after initiation of chondrogenic induction by TGF- $\beta 3$. Enlarged images of selected regions with yellowish, magenta, and red frames in $\mathbf{A}$ are respectively shown in $\mathbf{B}$. In these figures, representative stem cells are indicated by yellow arrows. Changes to the engineered tissues following chondrogenic induction of ECM production and PGA breakage are indicated by red and magenta arrows, respectively. Color images available online at www .liebertpub.com/ten.

multiphoton images were acquired from the "top side" of the scaffold. To assemble the small area images into a large area map, we used MetaMorph (Universal Imaging Corporation, Downingtown, PA). Because the cultured PGA scaffolds are not uniformly smooth specimens, the imaging depths we selected correspond to the sample plane in which a continuous distribution of the scaffold and cultured tissues are observed. In selecting the histological images for comparison to the multiphoton data, we chose the images in which the qualitative distribution of the scaffold and cultured tissues resemble that of the multiphoton images. These approaches were used because of the difficulty in defining and determining the exact depth of the imaging plane.

\section{RNA extraction and reverse transcriptase- polymerase chain reaction ( $R T-P C R)$}

Total RNA of the engineered tissues was extracted using Rezol reagent (Protech Technology, Taipei, Taiwan). One 
microgram of total RNA was reverse transcribed using random hexamers and reverse transcriptase (Promega, Madison, WI). The yielded first-strand cDNA was amplified by PCR using oligonucleotide primers for type I collagen (forward, 5'-CTTTGACCAACCGAACATGAC-3'; reverse, 5'-TTGAGCATTGCCTTTGATTGC-3'); type II collagen (forward, 5'-ACTTGCGTCTACCCCAATCC-3'; reverse, $5^{\prime}$-ACAGTCTTGCCCCACTTACC- $3^{\prime}$ ); and $\beta$ actin (forward, 5'-GAAATCGTGCGTGACATTAAG-3'; reverse, 5'-CTAGAAGCATTTGCGGTGGACGATGGAG GGGCC- $3^{\prime}$ ). The PCR protocol was an initial denaturation at $95^{\circ} \mathrm{C}$ for $3 \mathrm{~min}$ followed by 30 cycles of $94^{\circ} \mathrm{C}$ for $40 \mathrm{~s}, 55^{\circ} \mathrm{C}$ for $30 \mathrm{~s}$, and $72^{\circ} \mathrm{C}$ for $45 \mathrm{~s}$. PCR products were analyzed via electrophoresis in $2 \%$ agarose gels containing ethidium bromide, and visualized via a charge-coupled device (CCD) camera.

\section{Immunohistochemistry}

Immediately after multiphoton microscopic examination, the scaffolds were embedded in OCT compound at an orientation that their sectioning would begin from the "top sides." The cryostats were sectioned at 5- $\mu \mathrm{m}$ thickness and fixed in $4 \%$ paraformaldehyde for $5 \mathrm{~min}$ at $4^{\circ} \mathrm{C}$. The sections used for the following staining processes were at a depth of approximately 100-300 $\mu \mathrm{m}$ from the upper surface of the engineered tissues. Before immunolocalization of type I collagen, antigen retrieval was carried out in boiling $10 \mathrm{mM}$ citrate buffer solution ( $\mathrm{pH} 6.0$ ), and endogenous peroxidase activity was blocked with $3 \% \mathrm{H}_{2} \mathrm{O}_{2}$ in methanol for $20 \mathrm{~min}$. Nonspecific binding activities on the sections were then blocked with PBS containing $10 \%$ FBS and $0.2 \%$ goat serum. The sections were finally incubated with anticollagen I rabbit polyclonal antibody (Calbiochem, La Jolla, CA) at 1:50 dilution for $120 \mathrm{~min}$, followed by reaction with horseradish peroxidase (HRP)-conjugated goat antirabbit secondary antibodies (Chemicon, Temecula, CA) for $30 \mathrm{~min}$, and then chromogenically developed with 3amino-9-ethylcarbazole (AEC, DAKO, Carpinteria, CA) and counterstained with Mayer's hematoxylin (SigmaAldrich, St. Louis, MO). Before staining of type II collagen, the endogenous peroxidase activity was likewise blocked. To enhance antigen accessibility, the sections were incubated in $0.1 \mathrm{M}$ Tris-acetate, $\mathrm{pH} 7.6$, containing $40 \mathrm{mU} / \mathrm{mL}$ of chondroitinase and $1 \%$ bovine serum albumin (Sigma) at $37^{\circ} \mathrm{C}$ for $30 \mathrm{~min}$. Blocking serum (10\% FBS plus $0.2 \%$ rabbit serum) was used to block the nonspecific binding for $30 \mathrm{~min}$. The sections were then incubated with anticollagen II goat polyclonal antibodies (Santa Cruz Biotechnology, Santa Cruz, CA) at a 1:125 dilution for $120 \mathrm{~min}$, and rinsed with PBS. They were then incubated with HRP-conjugated rabbit antigoat secondary antibodies (Chemicon) for $30 \mathrm{~min}$. The immunoreactive areas were similarly developed with AEC and counterstained with Mayer's hematoxylin. Control slides were processed simi- larly except the step of primary antibody incubation was omitted.

\section{RESULTS}

\section{Multiphoton $A F$ and $S H G$ analysis of engineered tissues}

The multiphoton AF and SHG images of the engineered cartilage tissues acquired before (W0) and 1-3 weeks (W13) after chondrogenic induction are shown in Fig. 1. The large area multiphoton scans and the enlarged images from selected regions are shown in Fig. 1A and B, respectively. For the purpose of distinguishing the multiphoton $\mathrm{AF}$ and SHG signals, pseudocolors of green (AF) and blue (SHG) were assigned. In Fig. 1A, the combined AF and SHG images are shown in the right panel while the AF only results are shown on the left panel.

For the W0 sample, two morphological features are clearly visible under multiphoton imaging. The individual fibers of the PGA scaffold are strongly autofluorescent and are also second harmonic generating. The individual stem cells can also be identified through AF imaging. Because the DNA containing nuclei are known to be lacking in AF under multiphoton excitation, the stem cells appear as individual entities with the autofluorescent cytoplasm surrounding dim nuclei (yellow arrows in Fig. 1B).

One week after chondrogenic induction, multiphoton imaging reveals changes in the engineered tissues. In addition to the PGA scaffold fibers and stem cells, strongly second harmonic generating fibers start to be visible (red arrow). Because collagen has been shown to be effective generators of second harmonic signals, the appearance of the SHG fibers suggests the production of collagen-containing ECM. This observation is consistent with the production of collagen fibers revealed by immunohistochemistry. However, at this point, our multiphoton images do not allow a distinction between type I and II collagen to be made. In addition, the ECM fibers tend to align parallel to the neighboring PGA fibers, suggesting that the secreted collagen monomers use the PGA scaffold as a template for growth and such growth occurs parallel to the PGA fibers. In addition, the breakage in the PGA scaffold fibers starts to occur (magenta arrow). The implication in PGA fiber breakage becomes apparent when multiphoton autofluorescence and SHG images from $\mathrm{W} 2$ and $\mathrm{W} 3$ specimens are examined. For the $\mathrm{W} 2$ and $\mathrm{W} 3$ samples, in addition to the PGA fibers and proliferated stem cells, one notices several unique features. First, the ECM production greatly increased. In addition, the tendency for the ECM production to lie along the PGA fiber orientation tends to decrease as the ECM proliferates away from the PGA scaffold. As in the W1 specimen, we also noticed breaking points within individual PGA fibers. Combined with the qualitative observation that the PGA fibers become increasingly curvy with increasing 
chondrogenic induction time, our results suggest that associated with ECM production are scaffold structural alterations. Because the scaffolds remain well hydrated in culture, the most likely causes of the PGA structural alterations include mechanical force generation and biochemical degradation. These changes can lead to deformation of the PGA substrate to the extent of breaking individual fibers.

To add validity to the ability of multiphoton imaging in monitoring the physiological changes associated with engineered cartilage tissues, we also performed and compared the multiphoton results with those obtained using the conventional procedures of general observation, immunohistochemistry, and RT-PCR analysis.

\section{Gross appearance of engineered tissues}

The surface of the engineered tissues was rough at W0 and $\mathrm{W} 1$. The tissue surface became smoother and the cubic edges started to disappear at W2. The size of tissues shrank remarkably and the upper side became dome-shaped at W3 (Fig. 2). Multiphoton microscopy observation and tissue sectioning were consistently through the upper side. One key observation is the significant shrinkage of the W3 sample. This observation suggests that the ECM production generate internal forces that act on the PGA scaffold, leading to a volume reduction of engineered tissues. Such an observation is consistent with the multiphoton results described in the preceding text.

\section{Immunohistochemistry}

Immunoreactive type II collagen in the tissues was undetectable until 3 weeks of induction. On the W3 tissue, strong staining of type II collagen was clearly demonstrated in the ECM where chondrocyte-like cells were embedded (Fig. 3A).

Staining of type I collagen was in a different manner. Before induction, the fibroblastic MSCs showed positive staining of type I collagen in the cytoplasm. At this stage, only very scant ECM was noted. As early as 1 week after induction, ECM containing type I collagen was shown, and the intensity of staining was comparable through W1 to W3. Intriguingly, cytoplasmic expression of type I collagen decreased dramatically after 1 week of induction (Fig. 3B).

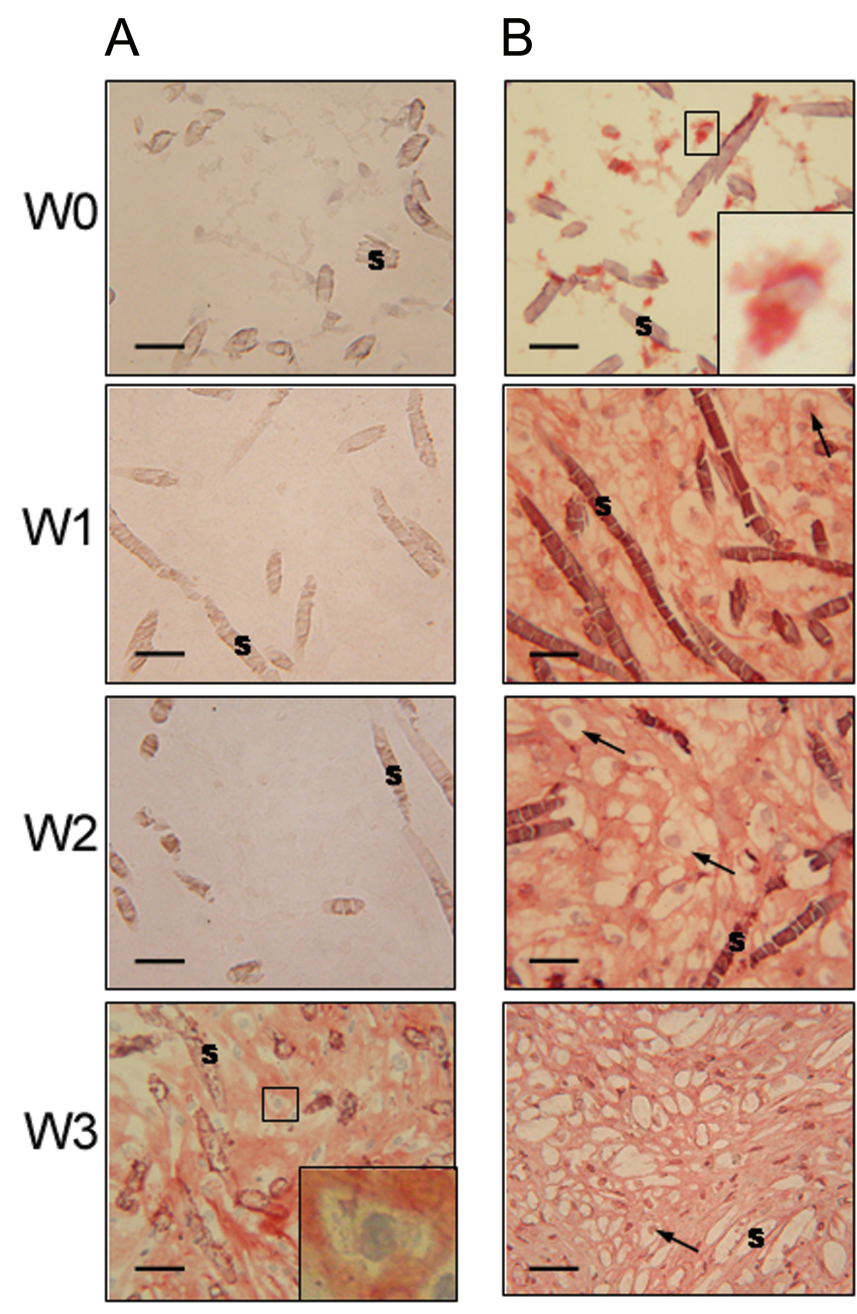

FIG. 3. Immunohistochemistry for localization of type II and type I collagens in the tissues. (A) Immunoreactive type II collagen was demonstrated only on the W3 tissue, localizing in the ECM. The inset in the W3 is the enlarged photo of the boxed area, showing a chondrocyte-like cell embedded in type II collagenpositive matrix. (B) The staining of type I collagen in the matrix of tissue W1, 2, and 3 was comparably intense. Interestingly, before formation of matrix in W0, the cytoplasm of MSCs was positively stained as shown in the inset in W0, which is the enlarged photo of the boxed area. As ECM was formed in W1-3, cytoplasmic staining became inconspicuous (arrows). PGA scaffolds $(\mathrm{S})$ were stained as background. Bars $=50 \mu \mathrm{m}$.
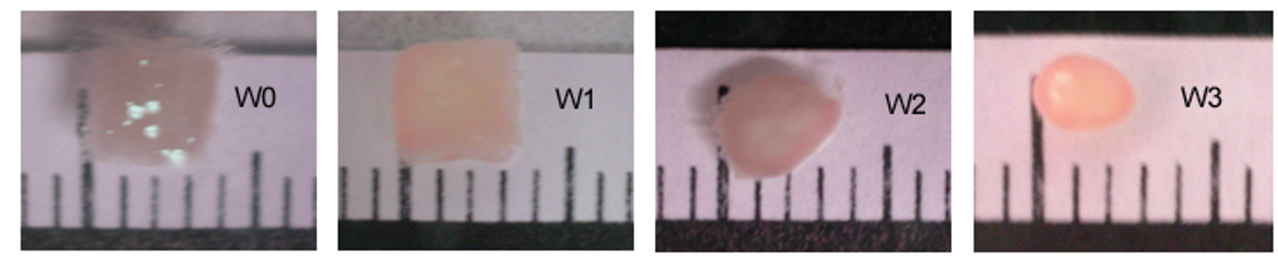

FIG. 2. Gross morphological changes of the engineered tissues (samples for RNA extraction). The surface of the tissues became smoother stepwise as culturing time increased. Note the size of the W3 sample shrank remarkably. Color images available online at www.liebertpub.com/ten. 


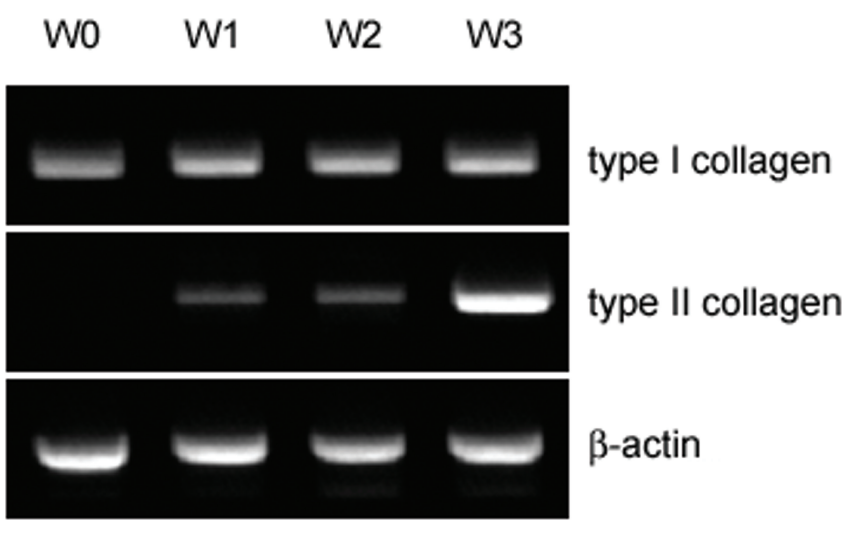

FIG. 4. RT-PCR showing the incremental expression of mRNA for type II collagen during the chronological periods of chondrogenic induction. In a different manner, the expression of type I collagen was constant throughout the course even before induction (W0). Amplification of $\beta$-actin serves as an internal loading control.

Similarly, ovoid-shaped chondrocyte-like cells were shown embedded in the matrix.

\section{$R T-P C R$}

Messenger RNA expression of type I and type II collagen in the engineered tissues was determined by RT-PCR analysis. The results (Fig. 4) did not show significant changes in the expression of type I collagen through W0 to W3, while that of type II collagen was minimally detectable at W1 and W2, and increased remarkably at W3.

\section{DISCUSSION}

The size of the engineered tissue shrank remarkably between 2 and 3 weeks of chondrogenic induction. The mechanisms for this size shrinking are unclear yet, but the images of ECM fiber orientation acquired using multiphoton microscopy suggest that the contraction of the newly formed matrix is most likely due to the internal forces associated with ECM production that acted on the PGA scaffold. Immunohistochemistry and RT-PCR showed evidence of cartilage-like tissue formation after 3 weeks of chondrogenic induction. The cells were in lacuna-like spaces embedded in the formed matrices. Expression of type II collagen in the matrix is indicative of chondrogenic differentiation.

A comparison of the RT-PCR results and the SHG imaging suggests that the increase in detected SHG signal corresponds to collagen production and our results indicate that ECM production can be monitored by direct multiphoton imaging without histological procedures. There are technical difficulties in ascertaining the observations via multiphoton microscopy, and via histology on the engineered tissue specimens were at the same orientation and depth. Nevertheless, the relative progressive amount and distribution of the newly formed matrix correlated very well between multiphoton microscopic and histological examinations. Another significant observation from multiphoton imaging is the breakage in the PGA scaffold associated with ECM production. Because one can also attribute such an observation under histological imaging to an artifact, the minimally invasive multiphoton observation eliminated doubts in establishing the fact that ECM production can lead to the generation of internal forces that result in the deformation and even damage of scaffold materials. To the best of our knowledge, this study is the first to image via multiphoton microscopy the progress of matrix production in the engineering of cartilage tissues. The key advantages associated with our approach are the ability to following morphogenesis of engineered tissues without addition of exogenous fluorophores and damaging histological procedures. Although confocal microscopy is widely applicable in many bioimaging applications, the reduced photodamage and enhanced imaging depths are the unique advantages associated with multiphoton microscopy. In addition, the SHG signal associated with collagen production and autofluorescence of the stem cells allow visualization without extrinsic labels. These advantages render multiphoton microscopy an invaluable technique for imaging tissue engineered constructs. In conclusion, multiphoton $\mathrm{AF}$ and SHG is capable of assessing the quality and yet retaining integrity of the engineered tissues before their implantation is established. With further development, this approach holds significant promise in understanding in vitro morphogenesis and following the growth of implanted engineered tissues under in vivo conditions.

\section{ACKNOWLEDGMENTS}

This work was supported by grants from National Science Councils, Executive Yuan, Taiwan, R.O.C. (to H-S.L. and C-Y.D.).

\section{REFERENCES}

1. Stock, U.A., and Vacanti, J. P. Tissue engineering: current state and prospects. Annu. Rev. Med. 52, 443, 2001.

2. Freed, L.E., and Vunjak-Novaskovic, G. Tissue culture bioreactors: chondrogenesis as a model system. In: Lanza, R.P., Langer, R., and Chick, W.L., eds. Principles of Tissue Engineering. San Diego: Academic Press, 1997, pp. 151-165.

3. Denk, W., Strickler, J.H., and Webb, W.W. 2-Photon laser scanning fluorescence microscopy. Science 248, 73, 1990.

4. So, P.T.C., Dong, C.Y., Masters, B.R., and Berland, K.M. Two-photon excitation fluorescence microscopy. Annu. Rev. Biomed. Eng. 2, 399, 2000.

5. Zipfel, W.R., Williams, R.M., Christie, R., Nikitin, A.Y., Hyman, B.T., and Webb, W.W. Live tissue intrinsic emission microscopy using multiphoton-excited native fluorescence 
and second harmonic generation. Proc. Natl. Acad. Sci. USA 100, 7075, 2003.

6. Zoumi, A., Yeh, A., and Tromberg, B.J. Imaging cells and extracellular matrix in vivo by using second-harmonic generation and two-photon excited fluorescence. Proc. Natl. Acad. Sci. USA 99, 11014, 2002.

7. Agarwal, A., Coleno, M.L., Wallace, V.P., Wu, W.Y., Sun, C.H., Tromberg, B.J., et al. Two-photon laser scanning microscopy of epithelial cell-modulated collagen density in engineered human lung tissue. Tissue Eng. 7, 191, 2001.

8. Powers, M.J., Janigian, D.M., Wack, K.E., Baker, C.S., Beer Stolz, D., and Griffith, L.G. Functional behavior of primary rat liver cells in a three-dimensional perfused microarray bioreactor. Tissue Eng. 8, 499, 2002.

9. Caplan, A.I., and Bruder, S.P. Mesenchymal stem cells: building blocks for molecular medicine in the 21 st century. Trends Mol. Med. 7, 259, 2001.

10. Pittenger, M.F., Mackay, A.M., Beck, S.C., Jaiswal, R.K., Douglas, R., Mosca, J.D., et al. Multilineage potential of adult human mesenchymal stem cells. Science 284, 143, 1999.

11. Li, W.J., Tuli, R., Huang, X., Laquerriere, P., and Tuan, R.S. A three-dimensional nanofibrous scaffold for cartilage tissue engineering using human mesenchymal stem cells. Biomaterials 26, 599, 2005.

12. Caterson, E.J., Nesti, L.J., Li, W.J., Danielson, K.G., Albert, T.J., Vaccaro, A.R., et al. Three-dimensional cartilage for- mation by bone marrow-derived cells seeded in polylactide/ alginate amalgam. J. Biomed. Mater. Res. 57, 394, 2001.

13. Lee, H.S., Huang, G.T., Chiang, H., Chiou, L.L., Chen, M.H., Hsieh C.H., et al. Multipotential mesenchymal stem cells from femoral bone marrow near the site of osteonecrosis. Stem Cells 21, 190, 2003.

Address reprint requests to: Hsuan-Shu Lee, M.D. Department of Internal Medicine National Taiwan University Hospital No. 7, Chung-Shan South Road Taipei, Taiwan 100

E-mail: benlee@ha.mc.ntu.edu.tw

or

Chen-Yuan Dong, Ph.D.

Department of Physics National Taiwan University No. 1, Sec. 4, Roosevelt Road Taipei, Taiwan 106

E-mail: cydong@phys.ntu.edu.tw 
